\title{
Competition between Populus euphratica and Tamarix ramosissima seedlings under simulated high groundwater availability
}

\author{
WU Guilin ${ }^{1,2}$, JIANG Shaowei ${ }^{1,2}$, LIU Weiyang ${ }^{3}$, ZHAO Chengyi ${ }^{1}$, LI Jun ${ }^{1 *}$ \\ ${ }^{1}$ State Key Laboratory of Desert and Oasis Ecology, Xinjiang Institute of Ecology and Geography, Chinese Academy of \\ Sciences, Urumqi 830011, China; \\ ${ }^{2}$ University of Chinese Academy of Sciences, Beijing 100049, China; \\ ${ }^{3}$ College of Plant Science, Tarim University, Alar 843300, China
}

\begin{abstract}
Desert riparian plants experience high variability in water availability due to hydrological fluctuations. How riparian plants can survive with low water availability has been well studied, however, little is known about the effects of high water availability on plant community structuring. We conducted a mesocosm experiment to test whether seedling competition under simulated high groundwater availability can explain the shift of co-dominance of Populus euphratica and Tamarix ramosissima in early communities to $P$. euphratica dominance in mature ones along the Tarim River in northwestern China. Seedlings of these two plant species were grown in monoculture and mixture pools with high groundwater availability. Results indicated that the above-ground biomass and relative yield of $T$. ramosissima were higher than those of $P$. euphratica. The competitive advantages of $T$. ramosissima included its rapid response in growth to groundwater enrichment and its water spender strategy, as evidenced by the increased leaf biomass proportion and the inert stomatal response to leaf-to-air vapor pressure deficit (VPD). In comparison, $P$. euphratica showed a conservative strategy in water use, with a sensitive response to leaf-to-air VPD. Result of the short-term competition was inconsistent with the long-term competition in fields, suggesting that competition exclusion is not the mechanism structuring the desert riparian plant communities. Thus, our research highlights the importance of mediation by environmental fluctuations (such as lessening competition induced by disturbance) in structuring plant communities along the Tarim riparian zones.
\end{abstract}

Keywords: community succession; vapor pressure deficit (VPD); stomatal conductance; riparian plant; water use strategy

Citation: WU Guilin, JIANG Shaowei, LIU Weiyang, ZHAO Chengyi, LI Jun. 2016. Competition between Populus euphratica and Tamarix ramosissima seedlings under simulated high groundwater availability. Journal of Arid Land, 8(2): 293-303. doi: 10.1007/s40333-015-0019-y

Riparian plants are subject to high variability in water availability due to hydrological fluctuations (Naiman and Décamps, 1997; Lytle and Poff, 2004; Stromberg et al., 2007; Stella et al., 2013). Such environmental variability is important for the dynamics of plant populations and community structuring (Hutchinson, 1961; Kotowski et al., 2010). Generally, environmental variability can shape communities through preventing the consistent effect of ecological interactions during harsh periods in which population growth is directly reduced and the intensity of interactions between organisms is lessened (Chesson and Huntly, 1997). Thus, in case of such variable riparian

*Corresponding author: LI Jun (E-mail: lijun@ms.xjb.ac.cn)

Received 2015-07-23; revised 2015-10-18; accepted 2015-10-22

(C) Xinjiang Institute of Ecology and Geography, Chinese Academy of Sciences, Science Press and Springer-Verlag Berlin Heidelberg 2016 
environments, while survivability during droughts is important for species success, competitive exclusion is expected to occur more likely during periods with high water availability under which plants are able to achieve rapid growth.

Riparian plant distribution has been shown to be jointly controlled by water availability and competition (Kotowski et al., 2010). Because competition takes place in the context of environmental conditions, competitive advantages need to be considered over a range of conditions (Sher and Marshall, 2003), especially in highly variable riparian habitats (Sabo et al., 2005). In desert riparian environments, for instance, species with the ability to grow rapidly in wet years and tolerate water deficit in drought years may be more competitive (Cleverly et al., 1997). Nevertheless, there have been extensive studies on the effects of flooding (Pollock et al., 1998; Vandersande et al., 2001; Tiegs et al., 2005; Corenblit et al., 2009) and drought (Gasith and Resh, 1999; Rood et al., 2000; Stella and Battales, 2010) on riparian plants, but little attention has been paid on the effects of favorable condition such as when groundwater is highly available.

Riparian plants are often established on newly deposited sediment near river channels after the recession of floods (Scott et al., 1996). As floodplain evolves through channel meandering or narrowing, riparian forests develop even-aged stands, with younger stands nearer to the channel (Stromberg, 1998; Friedman and Lee, 2002), suggesting that plant interactions at early stage play a critical role in the community structuring. In the Tarim Basin of northwestern China, the riparian forests, dominated by Populus euphratica Oliv. and sub-dominated by Tamarix ramosissima Ledeb. (Zhang et al., 2005), exhibit such a pattern that $P$. euphratica stand ages with distance from the active channel (Han et al., 2007). Commonly, co-dominance of P. euphratica and $T$. ramosissima in seedling stands shifts to P. euphratica dominance in mature ones (Fig. 1a) (Li et al., 2013). This dominance shift suggests that earlier space occupancy as a result of faster initial development of a species contributes to the species success (Körner et al., 2008). Specifically, $T$. ramosissima as a shrub species needs to suppress $P$. euphratica as a tree species at seedling stage, and vice versa, $P$. euphratica needs to occupy the riparian zones to avoid being suppressed by its co-established competitor at this stage. Seedling stands near channel are more exposed to hydrological fluctuations (Bottollier-Curtet et al., 2013), because the intensity and frequency of hydrological fluctuations decrease along the river-floodplain elevation gradient (Biswas and Mallik, 2010). In the upper reaches of the Tarim River Basin, riparian zones close to the active channel are still prone to naturally hydrological fluctuations that can result in an annual groundwater table variation greater than $2 \mathrm{~m}$ (Zhang et al., 2007). The subtle interspecific differences of riparian plants in response to such hydrological fluctuations during early succession may increase over time, and consequently determine the structure of the mature communities (Sher and Marshall, 2003). Therefore, the early competition between P. euphratica and $T$. ramosissima is expected to be an important process for structuring the riparian plant communities along the Tarim River.

Recent studies have shown that both $P$. euphratica and $T$. ramosissima are highly dependent on the groundwater availability, evidenced by leaf gas exchanges (Chen et al., 2011), sap flow (Ma et al., 2010), plant-water relations (Fu et al., 2006) and hydraulic conductance (Zhou et al., 2013). These works were conducted on adult plants, indicating that both species show little change in physiology when groundwater is relatively shallow (Chen et al., 2013). Results from another research by $\mathrm{Li}$ et al. (2013) indicated that, under shallow groundwater condition, both $P$. euphratica and $T$. ramosissima seedlings exhibited rapid shoot growth, whereas T. ramosissima accessed deeper groundwater more rapidly. These works focused on the effects of water availability on riparian plants at an individual level. Studies on the impact of water availability at a community level are entirely lacking. However, in riparian ecosystems in the southwestern USA with great success of Tamarix invasion (Glenn and Nagler, 2005), a field work indicated that the dominance of Tamarix mostly resulted from reduced competition intensity by native Populus and Salix due to groundwater decline and/or flood disappearance (Merritt and Poff, 2010). Controlled experiments also showed that these natives are competitive against Tamarix under favorable conditions (Sher et al., 2000; Sher and Marshall, 2003; Bhattacharjee et al., 2009). In addition, growth of native seedlings (Populus and Salix) impervious to Tamarix presence and density has 
been observed in fields (Sher et al., 2002). These evidences from the Tamarix invaded riparian ecosystems imply that $P$. euphratica may outcompete $T$. ramosissima under favorable conditions, a possible mechanism attributed to the success of $P$. euphratica along the Tarim riparian zones.

Our objective was to test whether the seedling competition between $P$. euphratica and $T$. ramosissima under high groundwater availability could explain the shift from $T$. ramosissima and $P$. euphratica co-dominance in early communities to $P$. euphratica dominance in mature ones. We also expected that the stronger competitor had higher water use capacity under high groundwater availability.

\section{Materials and methods}

\subsection{Materials}

The studied species, $P$. euphratica and T. ramosissima, are phreatophytic woody plants that dominate the riparian zones along the Tarim River in northwestern China. Both species exhibit life history strategies that adapt to riparian environments, such as producing tiny, wind- and water-dispersed seeds, releasing seeds from late summer to early autumn when flood events occur, and germinating on moist sediments after flood recessions. Their establishment niches overlap, leading to mixture seedling communities with similar dominance (Li et al., 2013). This situation means that the interspecific competition between them would be inevitable at seedling stage: the one that develops dense stands to occupy the riparian space earlier would be in a competitive advantage. Therefore, we used 3-year-old seedlings, the age when the canopy has not closed and neither species has developed dense stands in fields (Li et al., 2013).

Seeds of both species were collected in August 2011 from natural populations and then sown in a common garden at the Aksu Water Balance Station, Chinese Academy of Sciences $\left(40^{\circ} 27^{\prime} \mathrm{N}\right.$, $80^{\circ} 45^{\prime} \mathrm{E}$, hereafter Aksu Station), where the experiment was carried out. Then in March 2013, seedlings with heights ranging from 35 to $42 \mathrm{~cm}$ were transplanted into outdoor concrete pools designed for simulating different groundwater conditions.

\subsection{Experimental design}

The experimental pools were $2.8 \mathrm{~m} \times 1.8 \mathrm{~m}$ in area and $1.5 \mathrm{~m}$ in depth, with sealed bottoms and drainage valves at different depths. There was a coarse-sand layer (20-cm in thickness) at the bottom of each pool, above which loosely structured, loamy (sand:silt:clay averaged 25:70:5) soil that was collected from a riparian site was filled. In each pool a plastic pipe was vertically inserted for groundwater observation. There were total 10 pools gridded by concrete walls, seven for mixture to examine the competitive effects, two for $P$. euphratica monoculture and one for $T$. ramosissima monoculture as the reference for intraspecific effects. Seedlings were planted at the same density for all pools, with an inter- and between-row space of $0.4 \mathrm{~m}$, thus yielding 28 seedlings per pool. This density was similar to that of the mixture seedling communities at the same stage in fields. Species were alternated in space for mixture pools.

After transplantation, the bottom drainage valves were kept open, and weekly flood irrigations were carried out until the groundwater treatment initiation (on 1 July). In this way, soils in pools were kept moist while soil inundation was avoided, which promoted seedling establishment in the pools. From 1 July to 15 September (end of the experiment), drainage valves were closed and the groundwater was kept at $0.4-0.6 \mathrm{~m}$ below soil surface through flood irrigation. Groundwater depth in each pool was observed daily. Once the groundwater was close to $0.6 \mathrm{~m}$ in depth, water was added by flood irrigation. After each irrigation, if the groundwater was shallower than $0.4 \mathrm{~m}$, a drainage valve at this depth was opened to drain the excess water. Thus during the treatment period, the groundwater in each pool fluctuated with a small range. Here we only focused on the shallow groundwater condition because the work by Li et al. (2013), who compared the eco-physiology of the two species under a range of groundwater conditions, indicates that $P$. 
euphratica can likely compete against $T$. ramosissima at seedling stage only when groundwater is highly available. In addition, we have conducted a primary competition experiment (initiated in 2012), confirming that $P$. euphratica was excluded under the condition without groundwater availability (Fig. 1b).

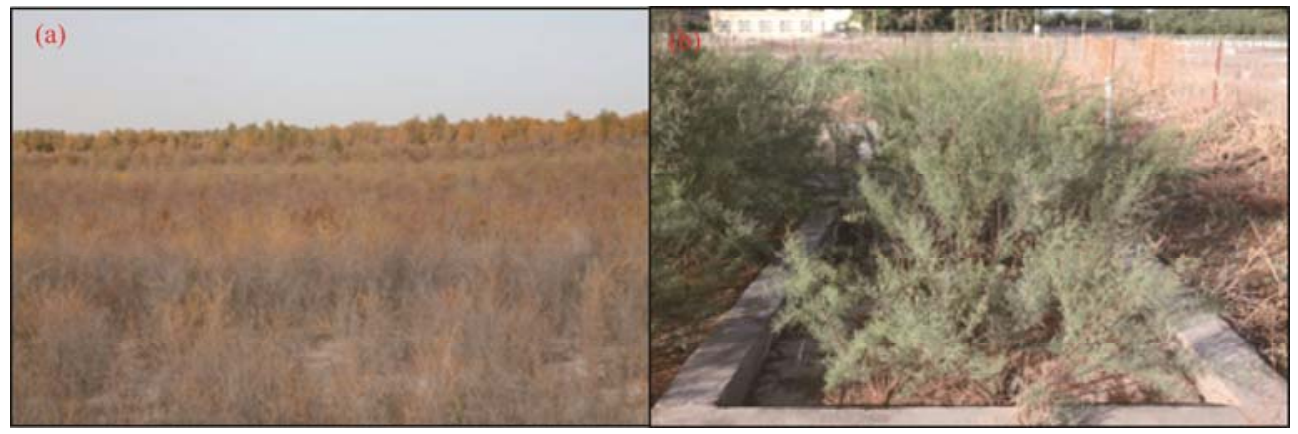

Fig. 1 Photographs showing plant communities in a riparian zone of the Tarim River (a) and a primary competition experiment conducted in a pool without groundwater availability (b). (a) Tamarix ramosissima (brown leaves) juveniles have been suppressed by those of Populus euphratica (yellow or green leaves) in the foreground in which there is similar abundance between the two species, and $P$. euphratica becomes dominant in the adult stand. (b) P. euphratica has been excluded from a pool without groundwater availability in the next year.

\subsection{Data collections}

As plant height is an important measure for competition effects, we numbered all seedlings in all pools and documented the changes in height for each seedling during the experiment. The initial heights were measured before the groundwater treatment. We had planned to measure the height monthly, but we observed that the seedlings grew rapidly under the experimental condition. Then from 22 July, the measurement was adjusted to be carried out weekly. At the end of the experiment, the above-ground biomass of each seedling was harvested and fresh biomass was immediately weighed. Then we randomly sampled 5 seedlings of each species from each mixture pool, 10 seedlings from each monoculture pool, of which leaves and branches were separated, and then weighed after being oven-dried to constant weight at $70^{\circ} \mathrm{C}$. We used the dry/fresh biomass ratio of each species in each pool to calculate dry above-ground biomass of each seedling in corresponding pools.

We determined plant water status by measuring pre-dawn $\left(\psi_{\mathrm{pd}}\right)$ and midday $\left(\psi_{\mathrm{md}}\right)$ xylem water potential with a PMS Model 1000 (PMS Instrument Co., Albany, USA) pressure chamber. Plant water status was measured twice, one on 14 August and the other on 12 September. Measurements were carried out between 05:30 and 06:30 for $\psi_{p d}$, local time, and between 15:30 and 16:30 for $\psi_{m d}$. For each time, plant water status was determined on 2 or 3 randomly selected seedlings in each monoculture pool, and 1 or 2 of each species in each mixture pool.

Gas exchange was measured by a portable photosynthesis system equipped with a $\mathrm{CO}_{2}$ injector (Li6400, Li-Cor, Lincoln, USA), with a standard chamber used for determining the stomatal responses to leaf-to-air vapor pressure deficit (VPD). Measurements were conducted on the youngest mature leaves in full exposure to sunlight. We carried out all of the leaf-gas exchange measurements between 10:30 and 13:30, local time, on clear days in August and early September, before which the seedlings' root systems had undergone enough time to acclimate to the water environment in pools. Leaf-to-air VPD-response measurements were performed on 3 randomly selected seedlings of each species in each mixture pool, and 7 in P. euphratica monoculture and 6 in $T$. ramosissima monoculture.

For determination of stomatal conductance $\left(g_{s}\right)$ in response to leaf-to-air VPD, we set the reference $\mathrm{CO}_{2}$ at $400 \mu \mathrm{mol} / \mathrm{mol}$, and the photosynthetic photon flux density (PPFD) at ambient level that ranged between 1,100 and $1,600 \mu \mathrm{mol} /\left(\mathrm{m}^{2} \cdot \mathrm{s}\right)$. We obtained within-cuvette leaf-to-air VPD gradient through controlling relative humidity $(\mathrm{RH})$, of which the values were targeted to the following levels in the order of $20 \%, 15 \%, 10 \%, 20 \%, 30 \%, 40 \%, 50 \%, 60 \%, 70 \%$ and $80 \%$. It should be noted that the cuvette $\mathrm{RH}>90 \%$ was difficult to be achieved due to low ambient air $\mathrm{RH}$ 
at the study site. This was also true for the cuvette $\mathrm{RH}<5 \%$ in cases of the seedlings with high leaf transpiration. During each measurement, we did not log the gas exchange parameters until $g_{s}$ had stabilized for 15-20 min. Subsequent measurement on each RH value was logged after $g_{s}$ had stabilized at least $2 \mathrm{~min}$. Leaves within the cuvette were cut immediately after the measurement, and then scanned for measuring the leaf area that was used to recalculate gas exchange parameters in the Li-Cor system.

\subsection{Data analysis}

The harvested seedlings were used to calculate the means for individual above-ground biomass and the leaf mass proportion to above-ground biomass for each species in each pool. Competitive effects were assessed with $t$-tests, in which the means of individual above-ground biomass from 7 mixture pools were compared with the means of the corresponding species from monoculture pools. The $t$-test was also used for determining the difference in leaf biomass allocation and plant water status. We used a competition index, termed relative yield (RY), expressed as the proportion of individual above-ground biomass of each species in mixture to that in monoculture, to evaluate the competitive effects of one species on the other (Weigelt and Jolliffe, 2003). RY difference between species was determined by ANOVA. Relative growth rate (RGR), calculated by $\mathrm{RGR}=(($ plant height at time $n+1)-($ height at time $n)) /($ height at time $n)$, was applied to characterize growth changes of both species in mixture or in monoculture. For detecting the sensitivity of $g_{s}$ to leaf-to-air VPD, we fitted the relationship between $g_{s}$ and ln-transformed leaf-to-air VPD with linear models, in which the higher slope indicates higher $g_{s}$ sensitivity. The difference in $g_{s}$ sensitivity was tested using analysis of covariance (ANCOVA). Leaf-level water use efficiency (WUE) was calculated by WUE $=P_{n} / T_{r}$, where $P_{n}$ is net photosynthesis rate and $T_{r}$ is transpiration rate. All statistical tests were performed using SPSS 13.0 (SPSS Inc., Chicago, IL, USA).

\section{Results}

\subsection{Competition effects}

Overall, the individual above-ground dry mass of $T$. ramosissima was several times greater than that of $P$. euphratica, regardless of in mixture or in monoculture (Fig. 2a). For P. euphratica, the individual above-ground dry mass was significantly greater $(P<0.01)$ in monoculture than in mixture, whereas an opposite pattern was found for $T$. ramosissima $(P<0.01)$. RY for $P$. euphratica, averaged 0.44 , was significantly $(P<0.01)$ lower than that of $T$. ramosissima, averaged 1.34, indicating that $T$. ramosissima was a stronger competitor in the experiment (Fig. $2 \mathrm{a}$ ). $T$. ramosissima also had significantly higher proportion of dry mass allocated to leaf than $P$. euphratica in both monoculture and mixture. Within species, $P$. euphratica showed no significant difference in leaf mass proportion between treatments, while T. ramosissima had a significantly higher value in mixture pools (Fig. 2b).
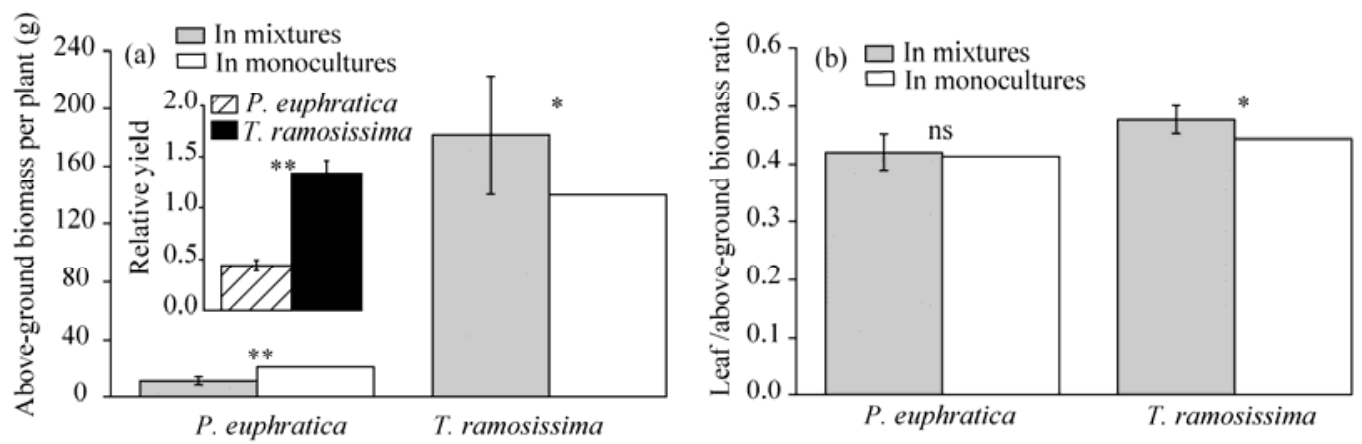

Fig. 2 The effect of competition on above-ground dry mass per plant (a) and leaf mass allocation (b) for $P$. euphratica and $T$. ramosissima in the experiment. Error bars indicate SE. An asterisk indicates significant difference within paired bars at $P<0.05$ level ( $t$-test), and double asterisks at $P<0.01$ level. The small panel in Fig. 2a indicates a significantly $(P<0.01)$ higher relative yield of $T$. ramosissima seedlings. 
The temporal patterns of RGR were distinct between species during the experiment (Fig. 3). T. ramosissima exhibited little difference in RGR between monoculture and mixture, with the value peaking in the first 3 weeks following treatment initiation, and then declining to a low level in the late stage of the experiment. In comparison, the RGR of $P$. euphratica increased with time following the treatment initiation, but exhibited an initial value greatly lower than that of $T$. ramosissima in the same stage. The RGR value for monoculture peaked one week later than for mixture, resulting in an extended period of higher growth rate for $P$. euphratica in monoculture.

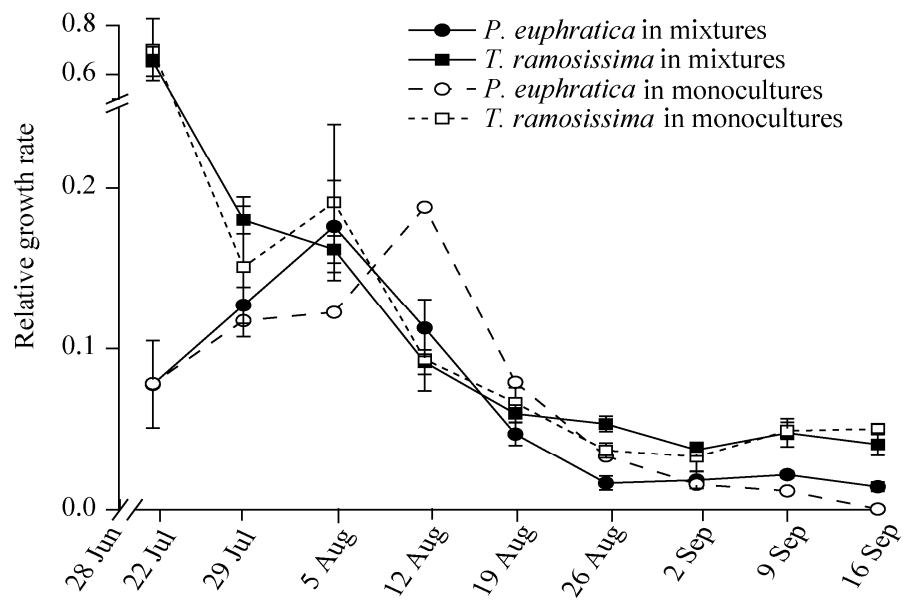

Fig. 3 Relative growth rate for P. euphratica and T. ramosissima in mixture and monoculture pools. Values are means $\pm \mathrm{SE}$ calculated from 7 mixture pools.

\subsection{Plant water status}

There was no difference either in $\psi_{p d}$ or $\psi_{m d}$ between monoculture and mixture for $P$. euphratica (Fig. 4). For T. ramosissima, monoculture seedlings displayed a significantly $(P<0.01)$ lower $\psi_{m d}$ but a similar $\psi_{p d}$ in comparison to mixture ones.

\subsection{Leaf gas exchange}

Net photosynthesis rate $\left(P_{n}\right)$ was higher in $P$. euphratica than in $T$. ramosissima throughout the obtained leaf-to-air VPD range, regardless of treatments (Fig. 5). As leaf-to-air VPD increased, the $P_{n}$ for $P$. euphratica seedlings in monoculture slightly decreased, while the $P_{n}$ for those in mixture tended to be constant. But there was no significant difference between treatments within the overlapped leaf-to-air VPD range. For $T$. ramosissima, the $P_{n}$ exhibited little fluctuation over the obtained leaf-to-air VPD range, with the seedlings in mixture having slightly higher values than those in monoculture.

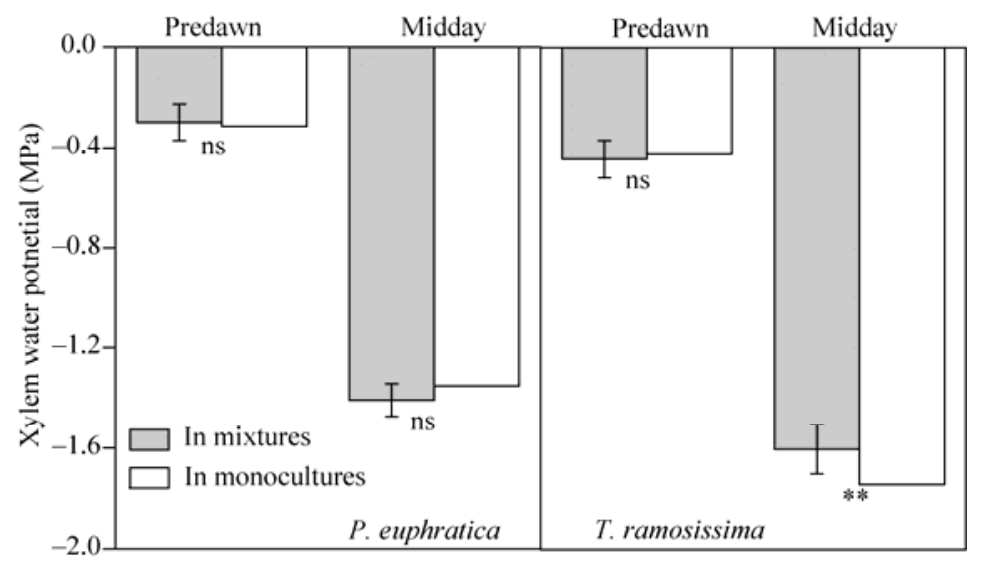

Fig. 4 Predawn and middayxylem water potential for $P$. euphratica and $T$. ramosissima seedlings in mixture and monoculture. Values are means \pm SE. Double asterisks indicate significant difference within paired bars at $P<0.01$ level and ns indicates no significant difference. 


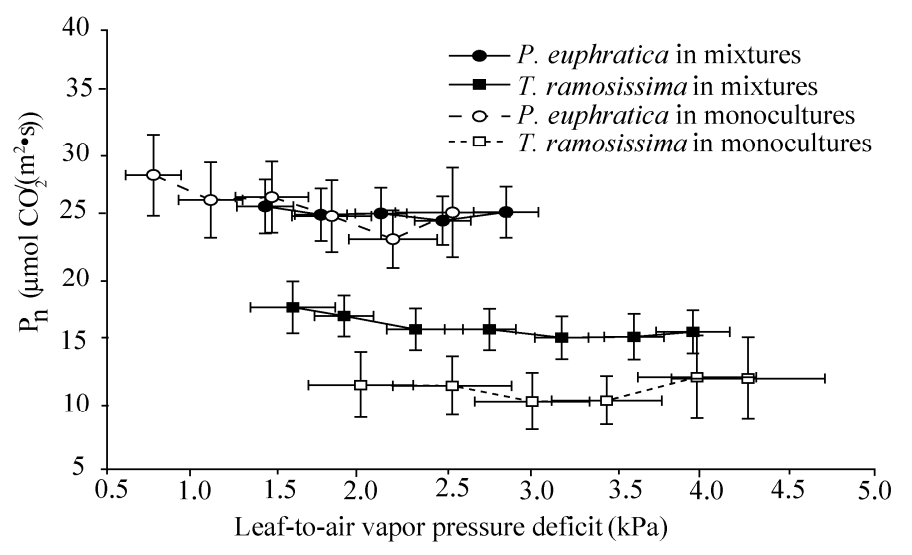

Fig. 5 Relationship between net photosynthetic rate $\left(\mathrm{P}_{\mathrm{n}}\right)$ and leaf-to-air vapor pressure deficit for $P$. euphratica and T. ramosissima in mixture and monoculture. Values are means $\pm \mathrm{SE}$.

For both species stomatal conductance $\left(g_{s}\right)$ declined linearly with the ln-transformed leaf-to-air VPD. Relationships between $g_{s}$ and $\ln \left(\right.$ leaf-to-air VPD) were strong $\left(R^{2}=0.80\right)$ for each species under each treatment (Fig. 6). The slope in the fitted models, as an indicator of the stomatal sensitivity to leaf-to-air VPD, differed between treatments for $P$. euphratica, with a significantly $(P<0.01)$ higher value in mixture than in monoculture $(0.78$ vs 0.44$)$. In comparison, it was similar between mixture and monoculture for T. ramosissima.

Leaf transpiration rate $\left(T_{r}\right)$ in both species increased in response to increasing leaf-to-air VPD. At a given leaf-to-air VPD, $P$. euphratica had a higher $T_{r}$ in monoculture than in mixture, while $T$. ramosissima showed an opposite pattern (Fig. 7a). Leaf water use efficiency (WUE) for both species declined rapidly in lower leaf-to-air VPD levels, and declined slightly over the higher values (Fig. 7b). For each species, WUE was greater in mixture than in monoculture, with the difference tending to be smaller with increasing leaf-to-air VPD.

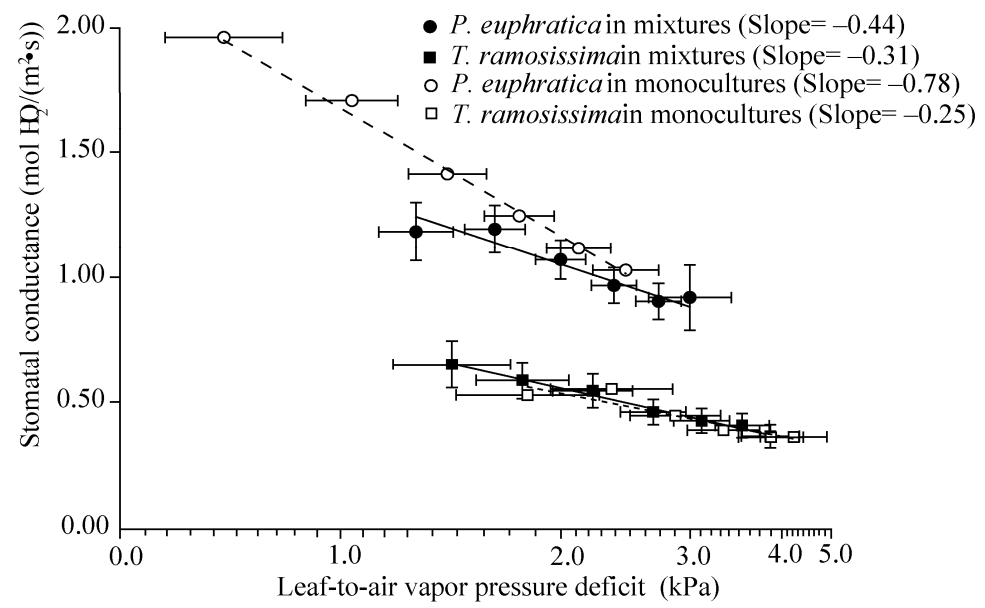

Fig. 6 Stomatal conductance in relation to leaf-to-air vapor pressure deficit for P. euphratica and $T$. ramosissima in mixture and monoculture. Slope of each fitted line represents stomatal sensitivity to leaf-to-air vapor pressure deficit. Values are means \pm SE. Note the $\ln$-transformed scale in $x$-axes.

\section{Discussion}

Although competition is considered important in structuring plant communities, it is difficult to predict the outcome of competitive interactions for many plant communities (Connell, 1983). This situation may be, for a given community, resulted from the lack of our understanding 

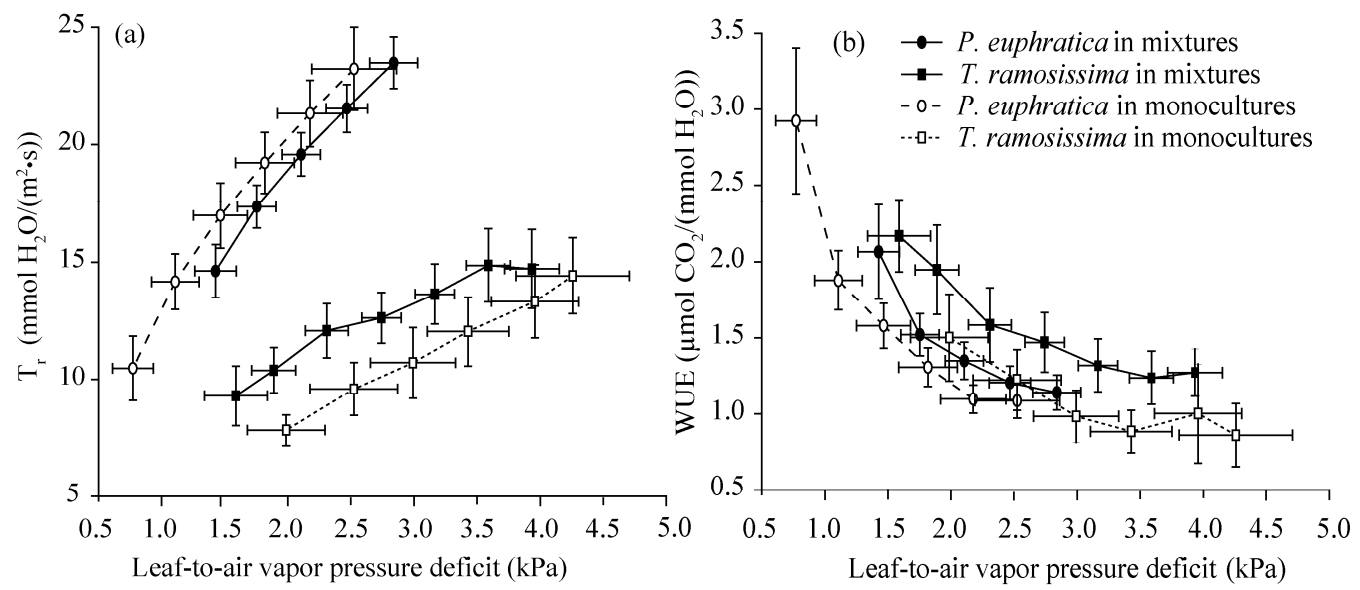

Fig. 7 Transpiration rate (a) and water use efficiency(WUE, b) responses to leaf-to-air vapor pressure deficit in $P$. euphratica and T. ramosissima. Values are means \pm SE.

in the role of competition: when, how and at which stage of life cycle of plants does competition determine community structure (Fowler, 1986). Indeed, this is true in case of our study, as $T$. ramosissima as the stronger competitor, evidenced by its higher RY (Fig. 2a), is contrary to the field observations. T. ramosissima exhibited a lower leaf-level transpiration (Fig. 7a) associated with a lower leaf-level $P_{n}$ (Fig. 5) and a similar WUE (Fig. 7b) compared to P. euphratica. However, the increased slope of the linear model between $g_{s}$ and ln-transformed leaf-to-air VPD in $P$. euphratica under intra-specific competition (Fig. 6) indicates that $P$. euphratica is more sensitive in stomatal response to leaf-to-air VPD increase. This suggests that, even with unlimited groundwater availability, $g_{s}$ would decrease at a larger degree to control water loss from leaves when air surrounding them becomes drier. Conversely, the inert stomatal response to leaf-to-air VPD in $T$. ramosissma allows the $g_{s}$ to decrease less under higher transpiration demand. Therefore, our expectation that the stronger competitor under high groundwater availability is associated with greater water use is largely supported. Overall, our results provide the strong evidences that the direct competition at early stage do not determine the structure of the late-successional communities along the Tarim riparian zones.

The competitive advantages of $T$. ramosissima over $P$. euphratica in our experiment involve several aspects in eco-physiology. Firstly, although with a lower leaf-level transpiration (Fig. 7a), $T$. ramosissima allocated higher proportion of biomass to leaf (Fig. 2b) in comparison to $P$. euphratica, indicating that $T$. ramosissima tends to increase its leaf area on the individual basis, a trait allowing fast growth for plants. These results are consistent with the findings from the $T$. ramosissima invaded riparian ecosystems in the southwestern USA, in which $T$. ramosissima has a comparable leaf-level water use but a greatly higher individual leaf area, and thus leading to the greater water use compared to the native phreatophytes (Sala et al., 1996). Secondly, in contrast to the sensitive stomatal response of $P$. euphratica to leaf-to-air VPD (Fig. 6), the inert stomatal response allow $T$. ramosissima seedlings to maintain relatively stable stomatal conductance during periods of high evaporative demand, as long as water availability for uptake is sufficient. Because stomata are controlled by the balance between water supply and loss, again, these results confirm that $T$. ramosissima is able to compensate water loss from leaves through uptake when groundwater is highly available (Sala et al., 1996), possibly due to an efficient hydraulic architecture. Inevitably, decrease in stomatal conductance in response to leaf-to-air VPD leads to decreased carbon gain (Ocheltree et al., 2013). In the long run, the optimal stomatal control is to maximize the carbon gain for given amount of water use (Buckley, 2005). Thus under the high water availability in our experiment, the inert stomatal response to leaf-to-air VPD as a water-spender strategy used by $T$. ramosissima has competitive advantage over the conservative water-use strategy by $P$. euphratica. Another important aspect is that $T$. ramosissima had a rapid growth in response to groundwater amelioration, as evidenced by its greater RGR than $P$. euphratica in the initial weeks following groundwater treatment (Fig. 3). To a large degree, this can be explained by the rapid root system responses of $T$. ramosissima to groundwater alterations (Li et al., 2013), which allows $T$. ramosissima to access to groundwater earlier than $P$. euphratica 
once groundwater depth is altered. Field observations also support that the growth of $P$. euphratica is more dependent on groundwater than that of T. ramosissima (Gries et al., 2003). Therefore, the stronger competitiveness of T. ramosissima in our experiment can be partly, if not fully, attributed to its inert stomatal response to leaf-to-air VPD, higher mass allocation to leaf and rapid response to groundwater alterations.

Stomatal sensitivity, an important role in the survival and success for a plant in competition, is affected by several environmental factors (Aasamaa and Sõber, 2011). Although the mechanism of stomatal response to leaf-to-air VPD has not been fully understood (Addington et al., 2003), the stomatal sensitivity to leaf-to-air VPD has shown to change considerably for plants experiencing soil water deficit at varying degrees (Bovard et al., 2005). Here, seedlings of both species in our experiment were grown under high groundwater, and the similar $\psi$ between treatments for $P$. euphratica (Fig. 4) suggests that the competition did not affect its water status. Thus, while the difference in stomatal sensitivity between species can be explained by species variation, the decreased stomatal sensitivity in mixture $P$. euphratica (Fig. 6) can not be explained by the plant water status. As hydraulic conductance increases with viscosity, a lower temperature resulting from shading would cause a decrease in hydraulic conductance for water movement to leaves (Mott and Peak, 2010). Under constant leaf-to-air VPD, $g_{s}$ increases in response to leaf temperature (Fredeen and Sage, 1999), consistent with the increased $T_{r}$ (Fig. 7a) and $g_{s}$ (Fig. 6) in $P$. euphratica in monoculture pools. Therefore, because stomatal sensitivity is related to a common spectrum of maximum $g_{s}$ (Ocheltree et al., 2013), the decreased stomatal sensitivity in $P$. euphratica in mixture may be caused by the shading by its competitor, which affects the whole-plant conductance through temperature alterations.

The unexpected outcome of our experimental competition could be related to our choice of young seedlings ( 3 years old): $P$. euphratica seedlings may need a few more years of growth to be more competitive. However, early competition from T. ramosissima is inevitable for $P$. euphratica due to the overlap of their establishment niches. Thus, how $P$. euphratica goes through the early competition against $T$. ramosissima becomes a key issue for understanding the early succession of the riparian plants along the Tarim River. Now it is known that T. ramosissima has both ruderal and stress tolerant characteristics (Glenn and Nagler, 2005), and, as a facultative phreatophyte, is able to use both groundwater and soil water (Busch and Smith, 1995). With these eco-physiological advantages, $T$. ramosissima is expected to be more competitive than $P$. euphratica in other conditions such that $P$. euphratica seedlings were excluded soon when no groundwater was available (Fig. 1b). Thus, our results suggest that explaining the success of $P$. euphratica should combine the integrative effects of environment fluctuations at a community level, which highlights the importance of the tradeoffs between costs and benefits of plant adaptions to long-term flow regimes (Lytle and Poff, 2004). For example, the higher biomass allocation to roots can allow plants to suffer less from flooding and drought, and enhance the ability to resprout at the cost of above-ground biomass reduction (Barrat-Segretain, 2001). Similarly, rapid growth may have the cost of more biomass loss during stressful periods such as drought and flooding (Sparks and Black, 1999; Rood et al., 2000). These tradeoffs suggest, from a perspective of long-term effects, that disturbances induced by hydrological fluctuations may favor $P$. euphratica more than $T$. ramosissima at early-successional stage through growth slowing, biomass losing or mortality, and thus weakening competition and preventing earlier space occupation by $T$. ramosissima. Hence the combined effects of varying hydrological conditions on the plant interaction are worthy of further work to warrant a general understanding of plant community structuring in riparian zones.

\section{Conclusions}

Our experiments show that $T$. ramosissima seedlings could exhibit competitive advantages over $P$. euphratica when groundwater is highly available. T. ramosissima benefits from its rapid responses to groundwater alterations, as well as a water-spender strategy including the inert stomatal response to leaf-to-air VPD and the higher mass allocation to leaf. Result of the experimental competition is inconsistent to the fact that $P$. euphratica is the final dominator in fields, suggesting that competition exclusion is not the mechanism for structuring the plant communities along the Tarim riparian zones. As seedling communities close to active channel suffer from disturbances 
imposed by hydrological fluctuations, the disturbance-induced competition lessening may favor $P$. euphratic going through the early competition against $T$. ramosissima. Our study highlights the importance of the mediation by environmental fluctuations in community structuring in riparian zones.

\section{Acknowledgments}

This research was funded by the National Basic Research Program of China (2013CB429903) and the National Natural Science Foundation of China $(41171037,41171095)$. The authors would like to thank TIAN Xiaohua and WANG Wenliang for their assistance during the experiment.

\section{References}

Aasamaa K, Sõber A. 2011. Stomatal sensitivities to changes in leaf water potential, air humidity, $\mathrm{CO}_{2}$ concentration and light intensity, and the effect of abscisic acid on the sensitivities in six temperate deciduous tree species. Environmental and Experimental Botany, 71(1): 72-78.

Addington R N, Mitchell R J, Oren R, et al. 2003. Stomatal sensitivity to vapor pressure deficit and its relationship to hydraulic conductance in Pinus palustris. Tree Physiology, 24(5): 561-569.

Barrat-Segretain M H. 2001. Biomass allocation in three macrophyte species in relation to the disturbance level of their habitat. Freshwater Biology, 46(7): 935-945.

Bhattacharjee J, Taylor Jr J P, Smith L M, et al. 2009. Seedling competition between native cottonwood and exotic saltcedar: implications for restoration. Biological Invasions, 11(8): 1777-1787.

Biswas S R, Mallik A U. 2010. Disturbance effects on species diversity and functional diversity in riparian and upland plant communities. Ecology, 91(1): 28-35.

Bottollier-Curtet M, Planty-Tabacchi A M, Tabacchi E. 2013. Competition between young exotic invasive and native dominant plant species: implications for invasions within riparian areas. Journal of Vegetation Science, 24(6): 1033-1042.

Bovard B D, Curtis P S, Vogel C S, et al. 2005. Environmental controls on sap flow in a northern hardwood forest. Tree Physiology, 25(1): 31-38.

Buckley T N. 2005. The control of stomata by water balance. New Phytologist, 168(2): 275-292.

Busch D E, Smith S D. 1995. Mechanisms associated with decline of woody species in riparian ecosystems of the Southwestern U.S. Ecological Monographs, 65(3): 347-370.

Chen Y N, Xu C C, Chen Y P, et al. 2013. Progress, challenges and prospects of eco-hydrological studies in the Tarim River Basin of Xinjiang, China. Environmental Management, 51(1): 138-153.

Chen Y P, Chen Y N, Xu C C, et al. 2011. Effects of groundwater depth on the gas exchange and chlorophyll fluorescence of Populus euphratica in the lower reaches of Tarim River. Acta Ecologica Sinica, 31(2): 344-353. (in Chinese)

Chesson P, Huntly N. 1997. The roles of harsh and fluctuating conditions in the dynamics of ecological communities. The American Naturalist, 150(5): 519-553.

Cleverly J R, Smith S D, Sala A, et al. 1997. Invasive capacity of Tamarix ramosissima in a Mojave Desert floodplain: the role of drought. Oecologia, 111(1): 12-18.

Connell J H. 1983. On the prevalence and relative importance of interspecific competition: evidence from field experiments. The American Naturalist, 122(5): 661-696.

Corenblit D, Steiger J, Gurnell A M, et al. 2009. Plants intertwine fluvial landform dynamics with ecological succession and natural selection: a niche construction perspective for riparian systems. Global Ecology and Biogeography, 18(4): 507-520.

Fowler N. 1986. The role of competition in plant communities in arid and semiarid regions. Annual Review of Ecology and Systematics, 17: 89-110.

Fredeen A L, Sage R F. 1999. Temperature and humidity effects on branchlet gas-exchange in white spruce: an explanation for the increase in transpiration with branchlet temperature. Trees, 14(3): 161-168.

Friedman J M, Lee V J. 2002. Extreme floods, channel change, and riparian forests along ephemeral streams. Ecological Monographs, 72(3): 409-425.

Fu A H, Chen Y N, Li W H. 2006. Analysis on water potential of Populus euphratica oliv and its meaning in the lower reaches of Tarim River, Xinjiang. Chinese Science Bulletin, 51(Suppl. 1): 221-228.

Gasith A, Resh V H. 1999. Streams in Mediterranean climate regions: abiotic influences and biotic responses to predictable seasonal events. Annual Review of Ecology and Systematics, 30: 51-81.

Glenn E P, Nagler P L. 2005. Comparative ecophysiology of Tamarix ramosissima and native trees in western U.S. riparian zones. Journal of Arid Environments, 61(3): 419-446.

Gries D, Zeng F, Foetzki A, et al. 2003. Growth and water relations of Tamarix ramosissima and Populus euphratica on Taklamakan desert dunes in relation to depth to a permanent water table. Plant, Cell and Environment, 26(5): 725-736. 
Han L, Wang H Z, Zhou Z L, et al. 2007. Population structure and demography of Populus euphraticu in upper and middle reaches of Tarim River. Acta Ecologica Sinica, 27(4): 1315-1322. (in Chinese)

Hutchinson G E. 1961. The paradox of the plankton. The American Naturalist, 95(882): 137-145.

Kotowski W, Beauchard O, Opdekamp W, et al. 2010. Waterlogging and canopy interact to control species recruitment in floodplains. Functional Ecology, 24(4): 918-926.

Körner C, Stöcklin J, Reuther-Thiébaud L, et al. 2008. Small differences in arrival time influence composition and productivity of plant communities. New Phytologist, 177(3): 698-705.

Li J, Yu B, Zhao C, et al. 2013. Physiological and morphological responses of Tamarix ramosissima and Populus euphratica to altered groundwater availability. Tree Physiology, 33(1): 57-68.

Lytle D A, Poff N L. 2004. Adaptation to natural flow regimes. Trends in Ecology and Evolution, 19(2): 94-100.

Ma J X, Chen Y N, Li W H, et al. 2010. Response of sap flow in Populus euphratica to changes in groundwater depth in the middle and lower reaches of the Tarim River of northwestern China. Chinese Journal of Plant Ecology, 34(8): 915-923. (in Chinese)

Merritt D M, Poff N L R. 2010. Shifting dominance of riparian Populus and Tamarix along gradients of flow alteration in western North American rivers. Ecological Applications, 20(1): 135-152.

Mott K A, Peak D. 2010. Stomatal responses to humidity and temperature in darkness. Plant, Cell and Environment, 33(7): 1084-1090.

Naiman R J, Décamps H. 1997. The ecology of interfaces: riparian zones. Annual Review of Ecology and Systematics, 28: $621-658$.

Ocheltree T W, Nippert J B, Prasad P V V. 2013. Stomatal responses to changes in vapor pressure deficit reflect tissue-specific differences in hydraulic conductance. Plant, Cell and Environment, 37(1): 132-139.

Pollock M M, Naiman R J, Hanley T A. 1998. Plant species richness in riparian wetlands-a test of biodiversity theory. Ecology, 79(1): 94-105.

Rood S B, Patiño S, Coombs K, et al. 2000. Branch sacrifice: cavitation-associated drought adaptation of riparian cottonwoods. Trees, 14(5): 248-257.

Sabo J L, Sponseller R, Dixon M, et al. 2005. Riparian zones increase regional species richness by harboring different, not more, species. Ecology, 86(1): 56-62.

Sala A, Smith S D, Devitt D A. 1996. Water use by Tamarix ramosissima and associated phreatophytes in a Mojave desert floodplain. Ecological Applications, 6(3): 888-898.

Scott M L, Friedman J M, Auble G T. 1996. Fluvial process and the establishment of bottomland trees. Geomorphology, 14(4): 327-339.

Sher A A, Marshall D L. 2003. Seedling competition between native Populus deltoides (Salicaceae) and exotic Tamarix ramosissima (Tamaricaceae) across water regimes and substrate types. American Journal of Botany, 90(3): 413-422.

Sher A A, Marshall D L, Taylor J P. 2002. Establishment patterns of native Populus and Salix in the presence of invasive nonnative Tamarix. Ecological Applications, 12(3): 760-772.

Sher A A, Marshall D L, Gilbert S A. 2000. Competition between native Populus deltoides and invasive Tamarix ramosissima and the implications for reestablishing flooding disturbance. Conservation Biology, 14(6): 1744-1754.

Sparks J P, Black R A. 1999. Regulation of water loss in populations of Populus trichocarpa: the role of stomatal control in preventing xylem cavitation. Tree Physiology, 19(7): 453-459.

Stella J C, Battles J J. 2010. How do riparian woody seedlings survive seasonal drought? Oecologia, 164(3): 579-590.

Stella J C, Rodríguez-González P M, Dufor S, et al. 2013. Riparian vegetation research in Mediterranean-climate regions: common patterns, ecological processes, and considerations for management. Hydrobiologia, 719(1): 291-315.

Stromberg J. 1998. Dynamics of Fremont cottonwood (Populus fremontii) and saltcedar (Tamarix chinensis) populations along the San Pedro River, Arizona. Journal of Arid Environments, 40(2): 133-155.

Stromberg J C, Lite S J, Marler R, et al. 2007. Altered stream-flow regimes and invasive plant species: the Tamarix case. Global Ecology and Biogeography, 16(3): 381-393.

Tiegs S D, O’Leary J F, Pohl M M, et al. 2005. Flood disturbance and riparian species diversity on the Colorado River Delta. Biodiversity and Conservation, 14(5): 1175-1194.

Vandersande M W, Glenn E P, Walworth J L. 2001. Tolerance of five riparian plants from the lower Colorado River to salinity drought and inundation. Journal of Arid Environments, 49(1): 147-159.

Weigelt A, Jolliffe P. 2003. Indices of plant competition. Journal of Ecology, 91(5): 707-720.

Zhang X W, Cheng T F, Chen H W, et al. 2007. Underground water monitoring and analysis on Tarim River Basin. Journal of Shihezi University: Natural Science, 25(3): 364-368. (in Chinese)

Zhang Y M, Chen Y N, Pan B R. 2005. Distribution and floristics of desert plant communities in the lower reaches of Tarim River, southern Xinjiang, People's Republic of China. Journal of Arid Environments, 63(4): 772-784.

Zhou H H, Chen Y N, Li W H, et al. 2013. Xylem hydraulic conductivity and embolism in riparian plants and their responses to drought stress in desert of Northwest China. Ecohydrology, 6(6): 984-993. 